\title{
Assessment of applying internal quality control and it is effect on accuracy of blood glucose measurement in some medical Laboratory in Kosti City, White Nile State, Sudan
}

\begin{abstract}
Quality control is essential in the clinical laboratory in order to ensure reliability of tests results, with acceptable levels of accuracy and precision. The impact of laboratory analysis in patient care is known as contributing to more than $70 \%$ of medical decisions. Internal quality control is an internal verification that the test yields day to day consistent results. The main objective of this study was to assess the effect of applying internal quality control on accuracy of test result in the clinical chemistry laboratories in Kosti City, Sudan. The study involved nine laboratories, stabilized control sera with known concentration (normal and pathological) was used to determine precision and accuracy. Structured questionnaires were also administered to assess the practice of internal quality control. Data were analyzed by using statistical package for social sciences (SPSS) version16. For validation of analytical process: means, standard deviations, coefficient of variations, accuracy and precision were obtained. The accuracy for the nine laboratories was $77 \%$ for both normal and pathological control sera and precision was $66.7 \%$. Coefficients of variations for seven laboratories were below $10 \%$. The study found that most of these laboratories do not follow the criteria of internal quality control associated with; calibration /recalibration instruments and pipettes $(66.7 \%)$, use of control sera $(55.6 \%)$, control charts $(77.8 \%)$ and SOPs $(77.8 \%)$. In conclusion, applying of internal quality control on clinical laboratory may help in the accuracy of test results, therefore, improving public health in developing countries.
\end{abstract}

Volume 8 Issue 3 - 2019

\author{
Osman Y Younis, ${ }^{1,2}$ Abdelhakam G Tamomh, ${ }^{3,4}$ \\ Mohammed A Suliman, ${ }^{3}$ Albadawi Abdelbagi \\ Talha, \\ 'Faculty of Medical Laboratory Sciences, University of Gezira, \\ Sudan \\ ${ }^{2}$ Department of Clinical Laboratory, Kosti Teaching Hospital, \\ Sudan \\ ${ }^{3}$ Faculty of Medical Laboratory Sciences, University of El Imam \\ El Mahdi, Sudan \\ ${ }^{4}$ College of Medical Laboratory, Dalian Medical University, China
}

Correspondence: Albadawi Abdelbagi Talha, Faculty of Medical Laboratory Sciences, University of Gezira, Wad Madani, Sudan, Emailbadawi@gmail.com

Received: March 26, 2019 | Published: May 17, 2019

Keywords: quality control, accuracy, pathological control sera, sudan

\section{Introduction}

Quality medical laboratory services provision is important in order to enhance diagnostic value and save life. This is because recognition of disease is the foundation of disease control and prevention. ${ }^{1}$ Resultantly there are variations in terms of laboratory services delivery from one country to another. In response to this challenge, in 1979, the world health assembly requested the world health organization (WHO) to develop a program for diagnosis in primary health care facilities in developing countries. ${ }^{2}$ Quality control programs are a means of controlling patient testing processes at the bench level. Laboratories must meet the established requirements for quality control of test methods; both the minimum required quality control $^{2}$ and any manufacturer's requirements must be followed. Quality laboratory service is an essential part of a health care delivery for diagnosis and monitoring of disease. But due to lack of awareness on the role of laboratory services in many developing countries, laboratory services have shortage of resource, poor management, ineffective services, ${ }^{3}$ low quality control measures, absence quality assurance programs, shortages of training and poor staff motivation. ${ }^{4.5}$ Achieving, maintaining and improving accuracy, timeliness and reliability are major challenges for health laboratories. Clinical chemistry laboratories are increasingly playing crucial roles in medicine as clinicians rely heavily on clinical chemistry tests to make proper diagnosis. Quality in clinical chemistry laboratories therefore cannot be compromised, especially in today's world where the emergence of sophisticated instrumentation and analytical methods has made laboratory tests so simple and easy to perform.
Such quality standards maintained by any laboratory if acceptable will not only benefit patients directly but also be an important boost for the laboratory. ${ }^{4} \mathrm{~A}$ standard requirement of all laboratories is to ensure the traceability of analytical results; something that cannot be achieved without good Internal quality control and external quality assessment practices. ${ }^{5}$ It is not surprising therefore that the international standards organization (ISO) has chosen both IQC and EQA as mandatory requirements for any laboratory rendering testing services. ${ }^{4,5}$

In 1981, the world health organization (WHO) used the term "internal quality control" (IQC), which it defined as "a set of procedures for continuously assessing laboratory work and the emergent results". IQC is an internal verification that the test yields consistent results day after day; in the other words, to identification measure of precision and accuracy. ISO15189 requires that "the laboratory shall design IQC systems that verify the attainment of the intended quality of results". IQC should be practiced periodically both during normal analytical runs and most importantly when a new method or equipment is to be used or tested. Simple IQC practices may include calibration, control and reference material measurements, within-run precision measurements, use of control charts, and use of simple statistics to interpret results. Precision and accuracy control are the main foci of IQC, their purpose are to ensure that analytical results are reliable, ${ }^{6}$ Simply, it is examining "control" materials of known substances along with patient samples to monitor the accuracy and precision of the complete analytic process. The unchallenged role played by analytical laboratory quality control is further made evident by considering the two main areas it encompasses: Internal Quality 
Control and External Quality Assessment. ${ }^{7}$ The use of statistical tools provides a visual means to understand quality control data so that timely action can be taken when method problems are detected. ${ }^{8}$

In the 1990 a popular view that surfaced was that laboratories should concentrate only on pre-analytical and post-analytical quality as analytical quality was no longer a problem. ${ }^{9}$ Describing this idea as a myth that was never true, westgard argues that to properly monitor and manage errors in the whole clinical laboratory testing process, it must start with analytical quality control. ${ }^{10}$ Used together, IQC and EQA provide a method of ensuring accuracy and consistency of results and are vital tools in the laboratory. EQA is an important complement to IQC in which a large number of laboratories are provided with the same material and required to return results to a coordinating centre, the results are compared to determine the accuracy of the individual laboratory. In addition, EQA provides continuous education and training for laboratories as well. EQA should, as far as possible, cover the entire range of tests, and the entire examination process, from sample reception, preparation and analysis to interpretation and reporting. ${ }^{8}$ EQA, also called internal laboratory comparison provides the perfect platform for the monitoring of laboratories, and it is a good marker of laboratory performance, competence and proficiency. Other goals of EQA include identification of internal laboratory differences, and assessment of how suitable a reference material may be for use in a test or procedure; all these aimed at establishing a betweenlaboratory and between-methods agreement. depending on the scope of the EQA scheme and the particular objectives chosen, one or more of four kinds of EQA studies can be employed: method evaluation, competence, certification and proficiency testing studies. ${ }^{11}$

In 2008 the world health organization regional office for Africa (WHO AFRO) introduces a stepwise accreditation approach to improving quality of laboratory in Africa. The program uses an incremental stepwise approach that is objectively measurable over time using international standards and the standards were adapted to the local environment. ${ }^{12}$

Avery recent publication by the WHO's African health monitor (2010) identified limited quality assurance and control protocol as one of the challenges faced by African clinical chemistry laboratories, and even proposed the establishment of a 'National Public Health Reference Laboratory' as a way out. poor quality control practices has been observed in our laboratories, as both IQC and EQA were very limited. ${ }^{13}$ In The present study we focus on assessment of applying and practice of internal quality control in laboratories by used known concentration control sera.

\section{Materials and methods}

\section{Study design and subject}

A descriptive, cross sectional-laboratory based study was conducted in nine medical laboratories selected according to ISO 15189 at Kosti City, ${ }^{14}$ during period of March 2017 to August 2017. Data were collected by using questionnaire which contain personal information and internal quality control information and a multi control sera, lyophilized serum of known concentration after preparation were divided into two aliquot containers, one labeled Normal (contain glucose target value $89.7 \mathrm{mg} / \mathrm{dl}$ ) and the second labeled Pathological (contain glucose target value $245 \mathrm{mg} / \mathrm{dl}$ ), then sent to laboratories to analyze for glucose levels (run as a samples). The nine medical laboratories were included in this study which received every day one aliquot container sample of control sera normal and one aliquot container of Pathological for fifteen days. The total number of samples were 270samples (135 Normal, 135 Pathological)

\section{Methodology}

The control sera is a lyophilized serum containing component concentrations suitable for quality control of the clinical laboratories, which known concentration for each analyzes in clinical laboratory test, for example glucose test to evaluate the instruments, reagents or pipettes which using in the lab, so the laboratories tests results compared by the known concentration (glucose $=89.7 \mathrm{mg} / \mathrm{dl} \& 245 \mathrm{mg}$ / dl) of control sera, according to the internal quality control procedure, Levey \& Jenning chart and Westgard Rules. ${ }^{15}$

\section{Multi control sera normal and pathological}

Intended use: Multi control sera are used in routine chemistry analyses quality control by monitoring accuracy and precision of mindray BS measurement system and test ability of clinical laboratory. Multi control sera (N \& P) are lyophilized control based on human serum $(5 \mathrm{ml})$. The concentrations or activities of control components are lot-specific and almost at abnormal or pathological level for the methods used.

\section{Preparation of multi control sera}

The multi control sera prepared according to Chosewood LC, in which the vial took out of the freezer and allowed to reaching room temperature. Tapped the vertically positioned vial gently and ensured that the lyophilized material was at the bottom of the vial. Then the screw cap and rubber removed carefully, avoiding any loss of lyophilizate. Reconstituted by adding $5.0 \mathrm{ml}$ distilled/deionized water to the side of the vial slowly and exactly. Then carefully replaced the rubber stopper and let it to stand for 30minutes at room temperature. Ensured that all components of lyophilizate were dissolved. The formation of foam avoided. Dispensed the required volume into sample cup, and then run QC test you have requested or have it analyzed in the same way as patient samples. ${ }^{16}$

Storage and stability: The control is stable up to expiration date indicated on the label when stored in unopened vial at $2 \sim 8^{\circ} \mathrm{C}$ and protected from light. Once reconstituted, please store control tightly capped when note in use. Once thawed to room temperature, the control not be re-freezen. ${ }^{16}$

Assay value: The control assay values (the target value and range) determined by mindray standard transfer procedure and routine method are listed in the value sheet. The target value was obtained from mindray measurement system and range was calculated as the target value $( \pm 3 \mathrm{SD})$ standard deviations. The control values are specific for different lot numbers and model of chemistry analyzers. ${ }^{16}$

Quality control interpretation: The control result must be within the defined range as shown in the control value sheet of the manufacturer. If the control result falls outside the range, the measurement system must be checked and corrective action must be taken or implemented.

Glucose method: Glucose oxidase- peroxidase (GOD) method according to manufacture in which the catalysis of glucose oxidase, glucose is oxidized to D-gluconate and hydrogen peroxide. Then at the present of peroxidase, a mixture of phenol and 4-Aminoantipyrine is oxidized to form a colored dye of quinineimine. The absorbency increase is directly proportional to the concentration of glucose and the procedure was performed firstly, by labeling three test tube as blank, test, and standard respectively, added $1 \mathrm{ml}$ for each tube 
from the glucose reagent, and added $10 \mu$ from target control sera (normal or pathological) to the test tube and $10 \mu 1$ from the standard reagent to the standard tube. Mixed and incubated for 10 minutes at $15-25^{\circ} \mathrm{C}$ and the absorbance reading and the glucose concentration Calculated as $(\mathrm{mg} / \mathrm{dl})$ by dividing the absorbance of a test sample with the absorbance of standard multiply by the factor hundred. ${ }^{17}$

Statistical analysis: Data were collected by using questionnaire which contains personal information, and internal quality control information. Then data were analyzed by using statistical package for social sciences (SPSS) version 16, and MS Excel 2010 (Redmond, USA) for windows. Mean, standard deviation, and coefficient of variation were calculated.

\section{Results and discussion}

This study was performed by using Multi control sera, lyophilized serum of known concentration, two levels of control sera Normal and Pathological, after preparation were divided into aliquot containers, labeled Normal (contain glucose target value $89.7 \mathrm{mg} / \mathrm{dl}$ ) and Pathological (contain glucose target value $245 \mathrm{mg} / \mathrm{dl}$ ). The populations for this study constituted nine laboratories were enrolled in this study Hospitals and Private laboratories in the medical laboratories field at Kosti City in Sudan, the control sera sent to laboratories daily for fifteen days to analyze for the one parameter glucose $(\mathrm{mg} / \mathrm{dl})$ run as a sample. The control sera is containing components concentrations suitable for quality control of the clinical laboratories, which known concentration for each analyte in clinical laboratory test, to evaluate the instrument, reagent or pipettes which using in the laboratory.

The laboratory results must be within the defined range as shown in the control value sheet of the manufacturer. Internal quality control is conducted by inserting control materials into every run of analysis. The results are plotted on control charts, If the laboratory results falls outside the range, the measurement system must be checked and corrective action must taken or implemented. So the results compared by the known concentration of control sera, according to the internal quality control procedure, Levey \& Jenning chart and Westgard rules were very important for acceptability of control data and determination of probability for rejection.

Multi control sera are used in routine chemistry analysis quality control for monitoring accuracy, precision and the performance of analytical methods.

Table 1 showed Head managers needed more training in quality control 55.6\%, and good applying for Management systems more than $77 \%$ in all laboratories selected in this study. But unfortunately all these laboratories showed poor applying to a quality manual book in the laboratory $88.9 \%$, and very poor applying for a sample rejection form in all laboratories were not found at all $(0 \%)$, also showed there were poor applying for quality indicators and Measures in the analytical phase in all laboratories selected in this study (77.8\%) did not used SOPs, and for internal quality control sample checked daily before any examination, just (44.4\%) used control sera, this problem similar finding conducted by Neumaier et al. ${ }^{18}$ and (77.8\%) not used control chart this similar to result in research conducted by Neumaier et al. ${ }^{18}$ Most of the laboratories were poor applying to quality for recalibration of pipettes after a period of operation (66.7\%) agree with Neumaier \& Afrifa ${ }^{18,19}$ and finding showed most of the laboratories used spectrophotometers $(77.8 \%)$ and only $(22.2 \%)$ used colorimeters (lab4 and lab6). Table 2 showed slightly variations between the laboratories Means and target value (mean $\pm \mathrm{SD}=89.7 \pm 4.5$ ) of normal control sera in all laboratories, But there were two laboratories lower than Target mean \pm SD (lab4 and lab6). Also showed means of pathological control sera (mean $\pm \mathrm{SD}=245 \pm 13$ ), laboratories were generally showed no variations in pathological control sera except (lab4) lower than Target mean $\pm \mathrm{SD}$. The medically allowable tolerances defined by the CLIA- 88 proficiency testing criteria for acceptable performance for glucose is $10 \%$. The parameters which demonstrated wide variation in the sigma (SD) values for both the levels of QC should be evaluated with discretion. The methodology should be re-evaluated. ${ }^{20}$ To assess CV\% in all laboratories, basis on this low $(\mathrm{CV} \%$ of $5 \%$ or less generally good method performance, whereas CVs of $10 \%$ and higher show unsatisfactory performance. ${ }^{21}$ Coefficients of variation (CV\%) for most of the glucose from the laboratories were below $10 \%$. except two laboratories in normal control (1ab4 and lab8) and in pathological control sera one lab (lab8) (Table 2). This finding means all laboratories generally good method performance. ${ }^{18,19}$ Table 3 showed a systemic error in all laboratories. The sign shows whether the test value is lower than the actual value. Accuracy is usually expressed as a percent difference, the laboratory results were said to be accurate if the difference between each reading and other did not exceed $\pm 2 \mathrm{SD}$, According to the Levey and Jenning chart of quality control. This fact explained that laboratories results were accurate test results within $\pm 2 \mathrm{SD}$, except one laboratory for both normal and pathological control sera (lab4); ${ }^{19}$ this may be here due to an improper calibration or mishandling of a measuring equipments (eg: pipettes). When evaluated the precision of test results of all nine laboratories, the laboratory results were said to be precise if the difference between each reading and other did not exceed $\pm 1 \mathrm{SD}$. So the better precision, the lower difference among the values, according to this fact three laboratories were not precise, this may be due to used colorimeters or Inadequate maintenance of the instrument that mains the quality of the instruments used play an important role in final result, so laboratory equipments used in clinical chemistry analysis is often plays a role in maintaining good accuracy and precision. This is expected there may be problems with both accuracy and precision in the measurements from these laboratories. ${ }^{22}$ The QC results are evaluated against various sorts of statistical QC rules, e.g., Westgard rules, which define specific performance limits and are designed to detect both random and systematic errors. The Westgard multi-rules are used to detect trends or shifts by examining individual values to determine the status of the measuring system. Westgard rules are based on sigma and are hence calculated without regard to constant sample sizes. These rules are commonly used with Levey-Jennings chart. ${ }^{23}$ Figures below showed highlights on laboratory errors based on Westgard multirules.

Figure 1 showed the Glucose results in days 4 and 15 exceeded $\pm 2 \mathrm{SD}$ (Rule:1/2s) warning rule, Patient results are acceptable also showed accurate and precision results for glucose test pathological control sera for lab1. Figure 2 showed 12 consecutive results from day 1 to the day 12; this is a "rejection rule," which is sensitive to systematic errors. Patient results are not acceptable and should be re-analyzed after corrective action has solved the problem. The following probable causes improper technique when handling the quality control, improper storage temperature correction of the quality control results and inadequate maintenance of the instrument. Also showed accuracy and precision results for glucose test pathological control sera for lab2. Figure 3 showed accuracy and precision results for glucose test normal control sera for lab3 and also the accuracy 
and precision results for glucose test pathological control sera for lab3. Figure 4 showed day 1,2,3,4,5,9,11,12,14 and 15 out of control (exceeded $\pm 3 \mathrm{SD}$ ), the results rejected indicates one control result has exceeded the established mean $+/-3 \mathrm{SD}$ range. This is a "rejection rule," which is sensitive to random error. Further corrective action should be conducted. Also showed day 1,2,6,7,10,11,12 and 14 exceeded -2SD, this is a "warning rule," day 9 result has exceeded3SD indicate an "out-of-control" condition, Patient results are rejected. A comprehensive check of the routine maintenance schedule and review sampling technique is recommended. Figure 5 showed 11 consecutive results have fallen on the same side of the mean this is a "rejection rule," which is sensitive to systematic errors. Patient results are not acceptable and should be re-analyzed after corrective action has solved the problem. Then showed day 2, 3, 4 and 5 exceeded \pm 2SD, control results has exceeded the established mean +/- 2SD range. This is a "warning rule," which does not indicate an "out-of-control" condition, but is intended to initiate further testing Patient results are acceptable, no corrective action is required. Day 1,6,7,8,9,10 and11 exceeded $\pm 1 \mathrm{SD}$. This is a "rejection rule," which is sensitive to systematic errors. A comprehensive check of the routine maintenance schedule and review sampling technique is recommended (Figure 6). Fifteen consecutive control results have fallen on the same side of the mean; this is a rejection rule which is sensitive to systemic errors. Patient results are not acceptable and should be re-analyzed after corrective action has solved the problem. The analyzer is out of control this may be an early indicator for (Shift) in the mean value. To resolve systemic error, corrective action should be conducted. Also showed day 6, 7 and 8 exceeded $\pm 2 \mathrm{SD}$, (Rule 1/2s) results has exceeded the established mean +/- 2SD range. This is a "warning rule," A comprehensive check of the routine maintenance schedule and review sampling technique is recommended. Day 9, 10, 11 and 12 four consecutive control results have exceeded the same mean +/- 1SD limit. This is a "rejection rule," which is sensitive to systematic errors. A systematic error exists. The analyzer is "out-of-control." This may be an early indicator for a "shift" in the mean value. Patient results are not acceptable and should be re-analyzed after corrective action has solved the problem. Figure 7 showed there were indicators for a "Trend" in the mean value. Patient results are not acceptable which indicate loss of reliability in the tests results and should be re-analyzed after corrective action has solved the problem. To resolve systematic errors, corrective action should be conducted; the probable causes may be due to: Aging of reagents or improper instrument calibration. Also showed day 1 exceeded \pm 2 SD warning rule ,day 2,3,4,8,9,11,12 and 13 exceeded \pm 1 SD a "rejection rule,". Figure 8 showed day 8 and 9 two tests results exceed the mean with a 4 standard deviation. This is a "rejection rule," which is sensitive to random error. The analyzer is "out-of-control." Patient results are not acceptable and should be re-analyzed after corrective action has solved the problem. Also showed day 8 and 11 exceeded \pm 3 SD rejection rule .Day 9 and 10 two tests results exceed the mean with a 4 standard deviation. This is a "rejection rule," Figure 9 showed accuracy results but imprecision. Also showed accuracy and precision results. Figure 10 showed there was systemic error in means of all nine laboratories in normal control sera (target mean=89.7) may be due to poorly calibrated/recalibrated pipettes or defect in use instrument standards or change in reagents batch. Then showed there was normal distribution of pathological control sera in all nine laboratories (target mean $=245$ ).

Table I Data analysis for managers of laboratories

\begin{tabular}{|c|c|c|c|}
\hline No & Variances & Yes & No \\
\hline I & Training courses in quality control & $44.40 \%$ & $55.60 \%$ \\
\hline 2 & Does the laboratory have an administrative structure? & $88.90 \%$ & $11.10 \%$ \\
\hline 3 & Does the laboratory have a way to evaluate employee performance? & $77.80 \%$ & $22.20 \%$ \\
\hline 4 & Is there a quality manual book in the laboratory? & $11.10 \%$ & $88.90 \%$ \\
\hline 5 & Is there an internal quality control program? & $66.70 \%$ & $33.30 \%$ \\
\hline 6 & Is there a team responsible for internal audit? & $66.70 \%$ & $33.30 \%$ \\
\hline 7 & Are corrective measures taken? & $77.80 \%$ & $22.20 \%$ \\
\hline 8 & $\begin{array}{l}\text { Is there a sample rejection form? Are the reasons for rejection clearly written and } \\
\text { displayed at the place where samples are received? }\end{array}$ & 0 & $100 \%$ \\
\hline 9 & Are SOPs for all tests done in the laboratory? & $22.20 \%$ & $77.80 \%$ \\
\hline 10 & Is the internal quality control sample checked daily before any examination & $44.40 \%$ & $55.60 \%$ \\
\hline II & Is there a Control chart (Internal Quality Control Table)? & $22.20 \%$ & $77.80 \%$ \\
\hline 12 & Are there documented procedures for the purchase of laboratory solutions? & $77.80 \%$ & $22.20 \%$ \\
\hline 13 & Is there a system to control the stock of reagents and laboratory solutions? & $77.80 \%$ & $22.20 \%$ \\
\hline 14 & Is the instrument calibrated when you buy? & $77.80 \%$ & $22.20 \%$ \\
\hline 15 & Is the "automatic pipette" calibrated upon purchase? & $55.60 \%$ & $44.40 \%$ \\
\hline 16 & Does the automatic pipette recalibrated after a period of operation? & $33.30 \%$ & $66.70 \%$ \\
\hline 17 & Type of instruments used in laboratories :colorimeter & $22.20 \%$ & $77.80 \%$ \\
\hline 18 & Type of instruments used in laboratories :spectrophotometer & $77.80 \%$ & $22.20 \%$ \\
\hline
\end{tabular}


Table 2 The Lab mean, SD and CV\% for both Normal and pathological control sera

\begin{tabular}{|c|c|c|c|c|c|c|c|c|}
\hline & \multicolumn{4}{|c|}{ Normal control sera } & \multicolumn{4}{|c|}{ Pathological control sera } \\
\hline & $\begin{array}{l}\text { Lab } \\
\text { mean }\end{array}$ & Lab SD & $\begin{array}{l}\text { Target } \\
\text { mean } \pm S D\end{array}$ & CV\% & Lab mean & Lab SD & $\begin{array}{l}\text { Target } \\
\text { mean } \pm S D\end{array}$ & CV\% \\
\hline Labl & 89.7 & 4.78 & & $5.3 \%$ & 249.3 & 7 & & $2.8 \%$ \\
\hline Lab2 & 85.7 & 2.6 & & $3 \%$ & 241.2 & 10 & & $4.1 \%$ \\
\hline Lab3 & 87.5 & 3.2 & & $3.7 \%$ & 247.5 & 5.1 & & $2.1 \%$ \\
\hline Lab4 & 76.2 & 8.4 & & $11 \%$ & 215.6 & 6 & & $2.8 \%$ \\
\hline Lab5 & 86.1 & 3.2 & & $3.7 \%$ & 249.5 & 22.8 & & $9.1 \%$ \\
\hline Lab6 & 82.7 & 2.8 & $89.7 \pm 4.5$ & $3.4 \%$ & 234.5 & 12.4 & $245 \pm 13$ & $5.3 \%$ \\
\hline Lab7 & 88.4 & 7.56 & & $8.6 \%$ & 250.2 & 18.1 & & $7.2 \%$ \\
\hline Lab8 & 86.1 & 8.99 & & $10.4 \%$ & 242.1 & 30.3 & & $12.5 \%$ \\
\hline Lab9 & 87.4 & 2.8 & & $3.2 \%$ & 242.6 & 6.7 & & $2.8 \%$ \\
\hline
\end{tabular}

Table 3The Accuracy and precision for both Normal and pathological control sera

\begin{tabular}{|c|c|c|c|c|}
\hline \multirow[b]{2}{*}{ Labs } & \multicolumn{2}{|c|}{$\begin{array}{l}\text { Normal control sera } \\
\text { (glucose test) }\end{array}$} & \multicolumn{2}{|c|}{$\begin{array}{l}\text { Pathological control sera } \\
\text { (glucose test) }\end{array}$} \\
\hline & $\begin{array}{l}\text { Accuracy } \\
\text { (difference \%) }\end{array}$ & $\begin{array}{l}\text { Precision } \\
\text { (difference ) }\end{array}$ & $\begin{array}{l}\text { Accuracy (difference } \\
\%)\end{array}$ & Precision (difference ) \\
\hline $\operatorname{Lab}(I)$ & $0 \%$ & 0 & $1.7 \%$ & 4.3 \\
\hline $\operatorname{Lab}(2)$ & $-4.8 \%$ & -4.3 & $-1.8 \%$ & -4.5 \\
\hline $\operatorname{Lab}(3)$ & $-2.5 \%$ & -2.2 & $0.98 \%$ & 2.4 \\
\hline $\operatorname{Lab}(4)$ & $-15 \%$ & -13.5 & $-12 \%$ & -29.4 \\
\hline $\operatorname{Lab}(5)$ & $-4.1 \%$ & -3.7 & $1.8 \%$ & 4.5 \\
\hline $\operatorname{Lab}(6)$ & $-7.8 \%$ & -7 & $-4.1 \%$ & -10 \\
\hline $\operatorname{Lab}(7)$ & $-1.4 \%$ & -1.3 & $2.1 \%$ & 5.2 \\
\hline $\operatorname{Lab}(8)$ & $-6.2 \%$ & -5.6 & $-1.2 \%$ & -2.9 \\
\hline Lab(9) & $-2.6 \%$ & -2.3 & $-0.98 \%$ & -2.4 \\
\hline
\end{tabular}

A

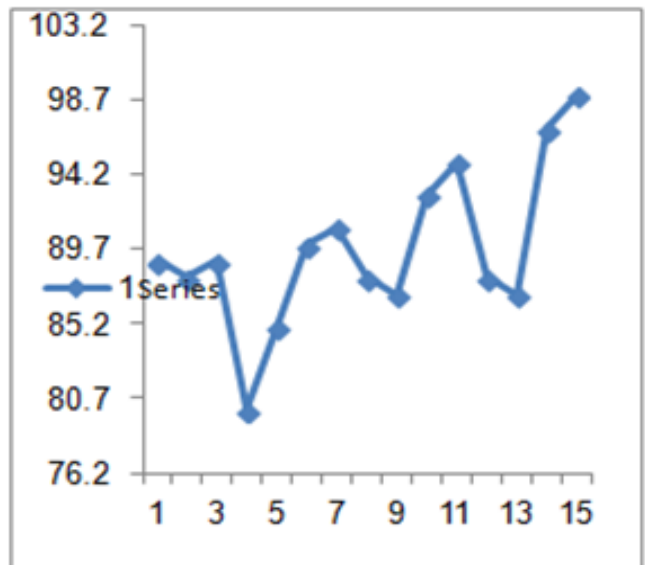

B



Figure I labl results for glucose test (A) normal control sera (B) pathological control sera 
A

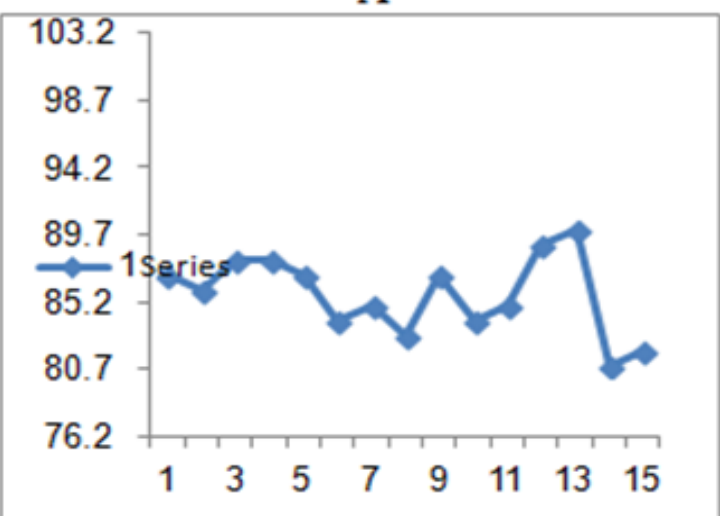

B

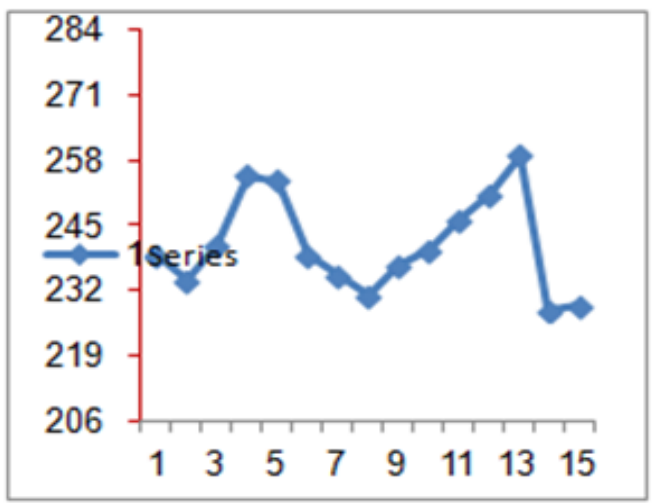

Figure 2 lab2 results for glucose test test (A) normal control sera (B) pathological control sera.

A

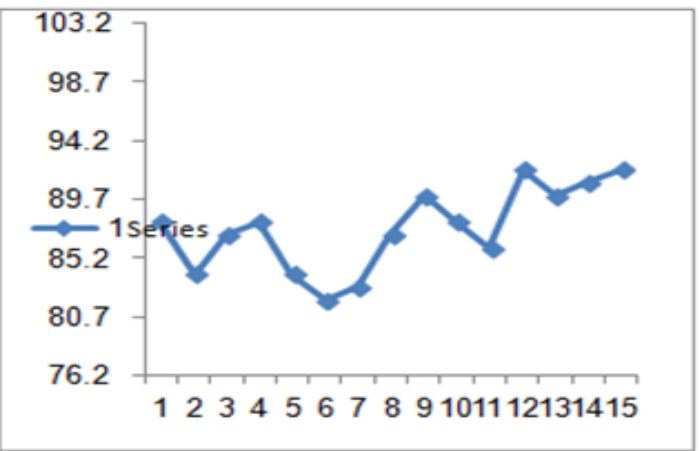

B

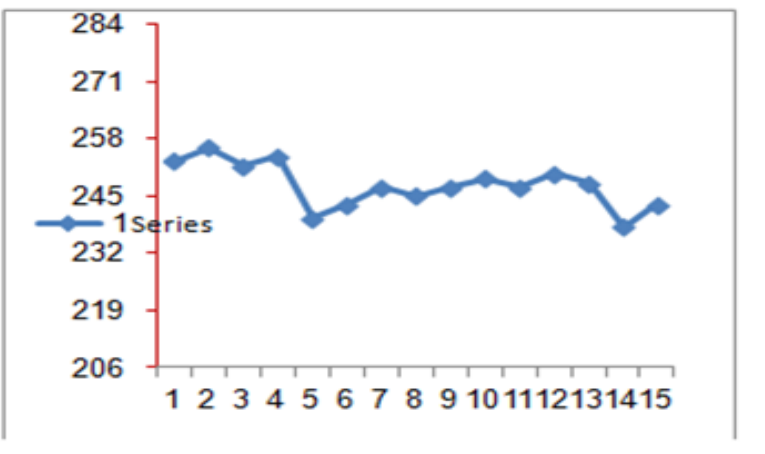

Figure 3 lab3 results for glucose test (A) normal control sera (B) pathological control sera.

A

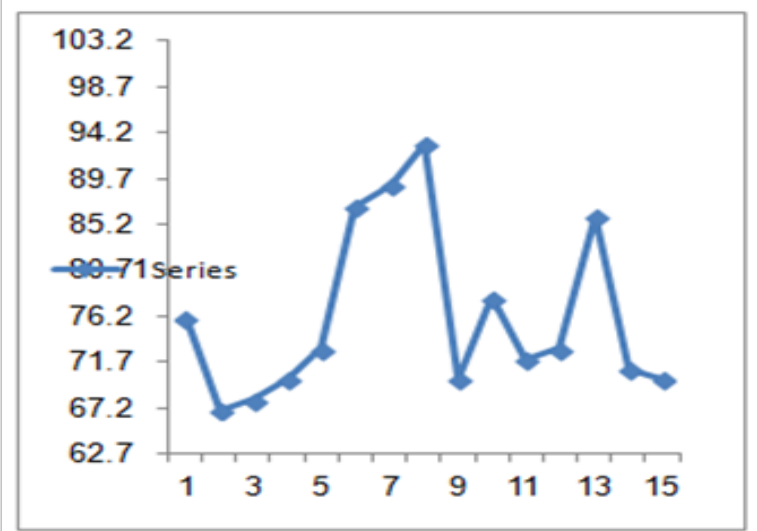

B



Figure 4 lab4 results for glucose test (A) normal control sera (B) pathological control sera. 




Figure 5 lab5 results for glucose test (A) normal control sera (B) pathological control sera.

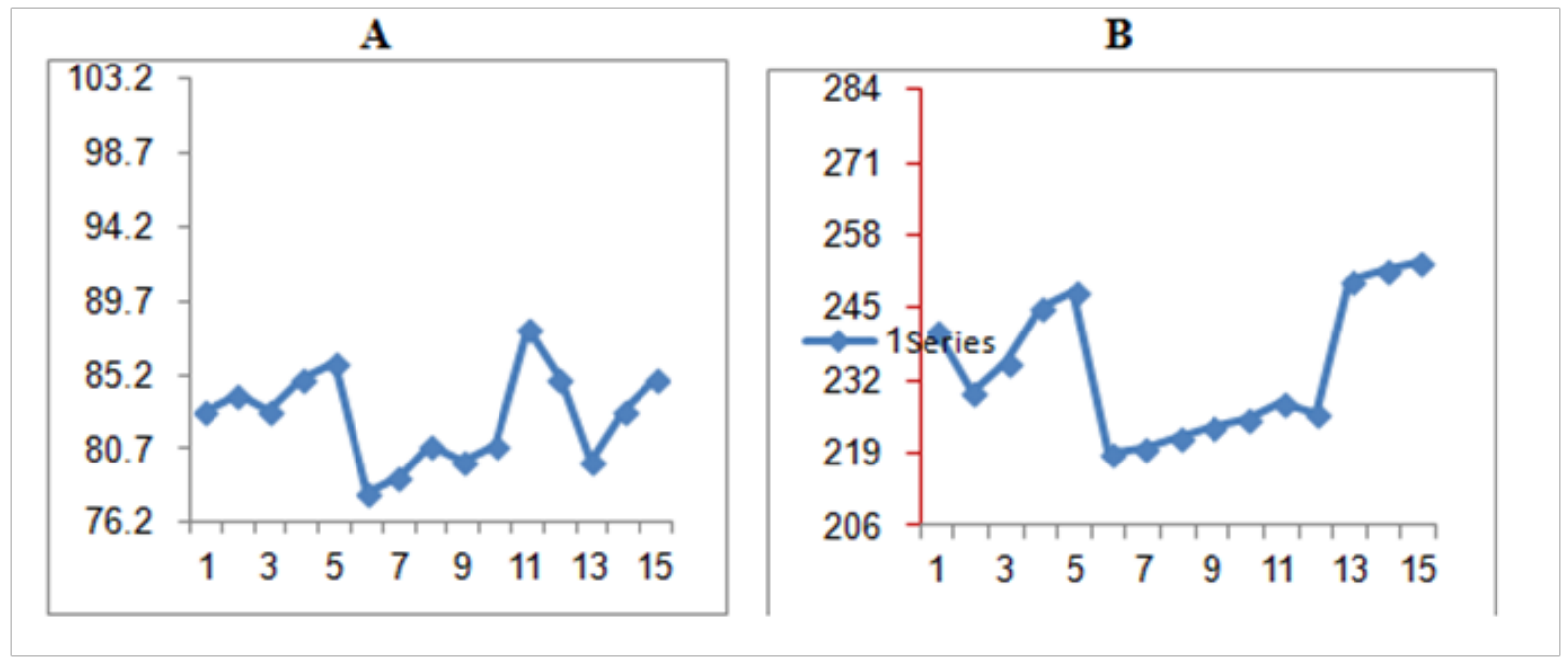

Figure 6 lab 6 results for Glucose test (A) normal control sera (B) pathological control sera.

A

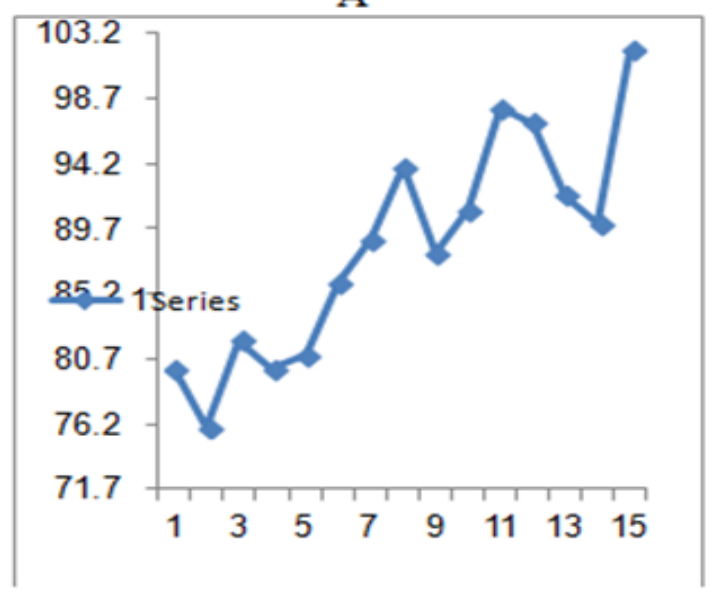

B

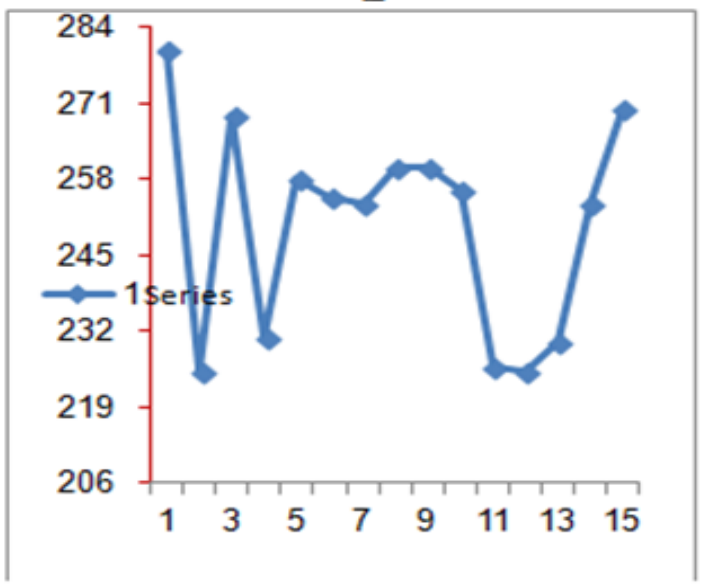

Figure 7 lab7 results for glucose test (A) normal control sera (B) pathological control sera. 
A

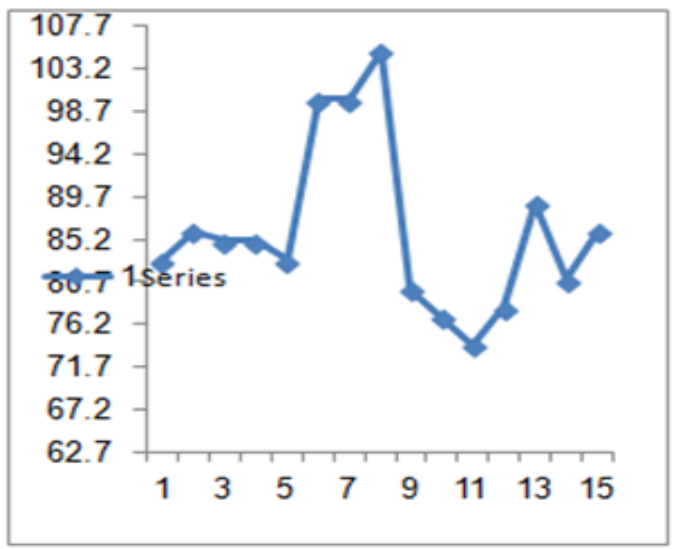

B

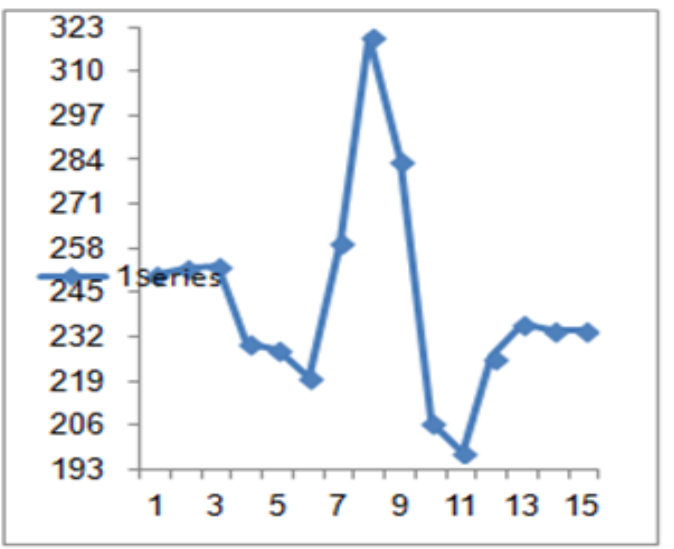

Figure 8 lab8 results for glucose test (A) normal control sera (B) pathological control sera.

A

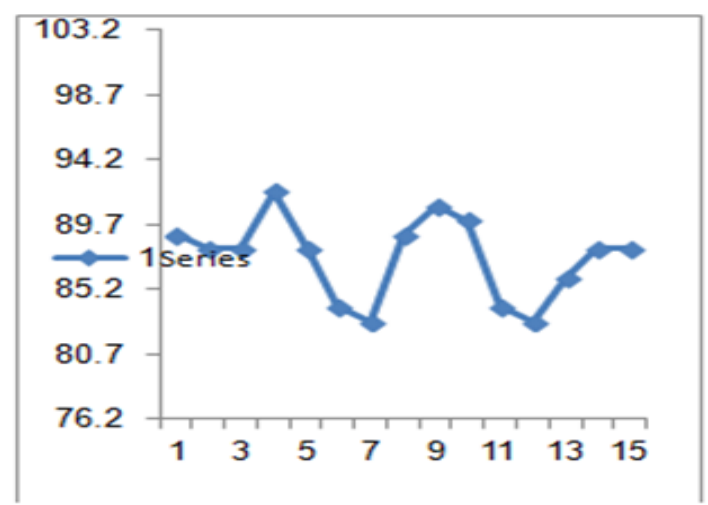

B

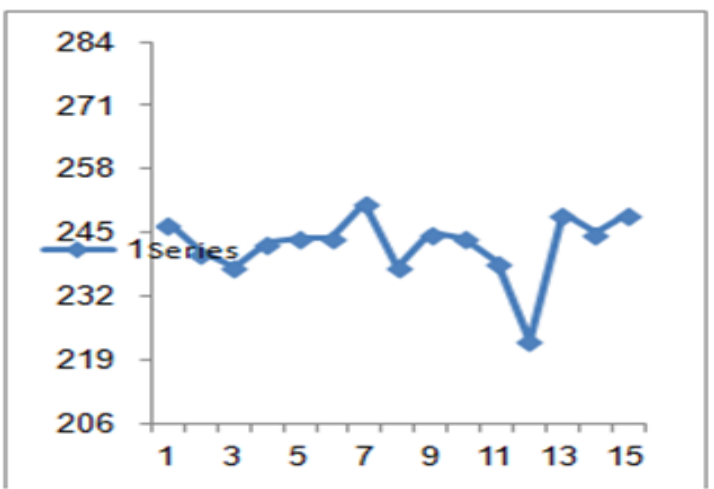

Figure 9 lab9 results for glucose test (A) normal control sera (B) pathological control sera.

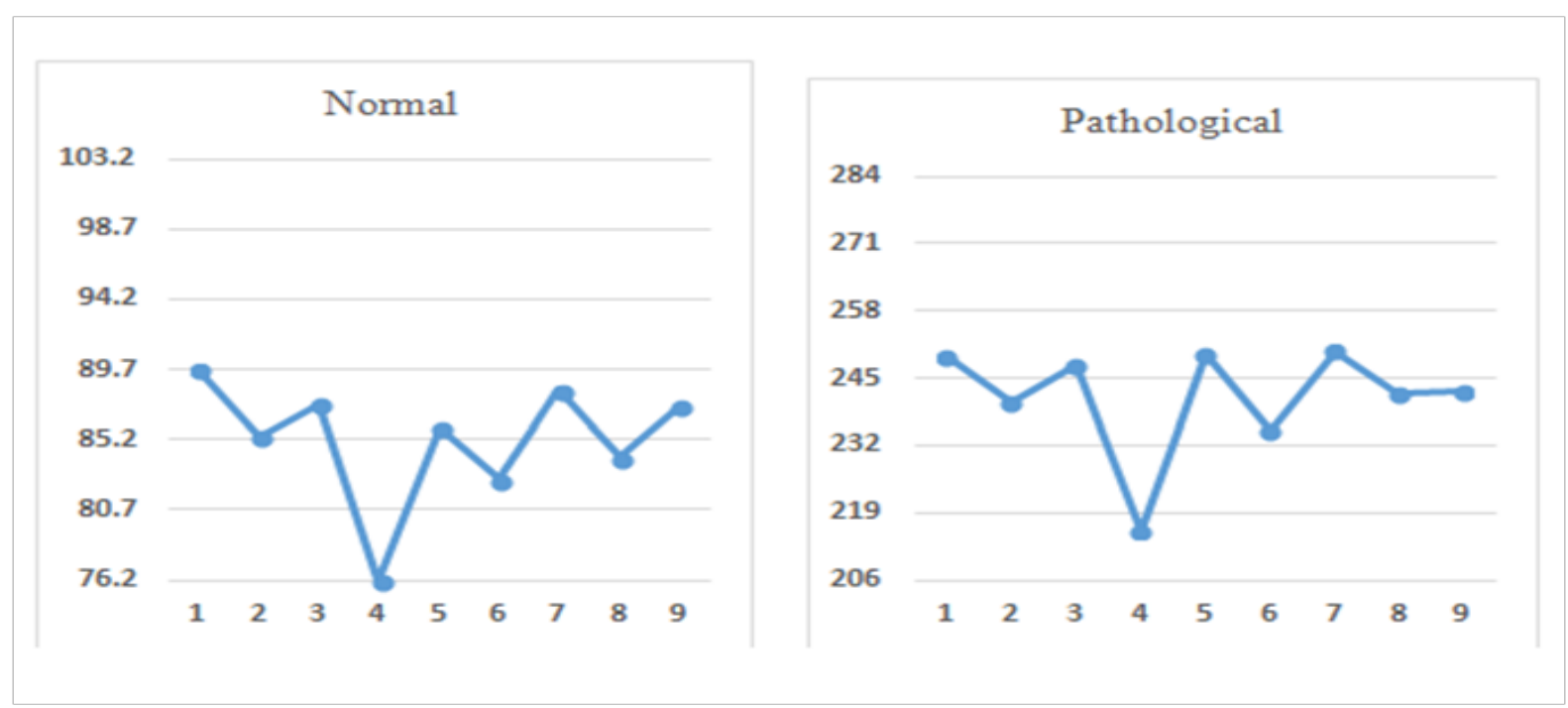

Figure I 0 Distribution of means for all nine laboratories to normal and pathological control sera. 


\section{Conclusion}

The evaluation and assessment of applying and practice of internal quality control in laboratories by using known concentration of control sera was conducted. The application of internal quality control in clinical laboratory may help in the accuracy and reliability of test results, therefore, a better diagnosis and treatment, and improving public health in developing countries. Further studies should be conducted in the study area, and applying of control sera, control Charts and SOPs must be use mandatory in each laboratory in Kosti City.

\section{Acknowledgments}

None.

\section{Conflicts of interest}

Author declares there is no conflict of interest.

\section{References}

1. Saliki JT. The role of diagnostic laboratories in disease control. Ann NY acad Sci. 2000;916:134-138.

2. Koplan JP, Puska P, Jousilahti P, et al. Improving the world's health through national public health institutes. Bull World Health Organ. 2005;83(2):154-157.

3. Dacombe RJ, Squire SB, Ramsay AR, et al. Essential medical laboratory services: their role in delivering equitable health care in Malawi. Malawi Medical Journal. 2006;18(2):77-79.

4. Schroeder LF, Amukele T. Medical laboratories in sub-Saharan Africa that meet international quality standards. Am J Clin Pathol. 2014;141(6):791-795

5. Cooper WG. Quality control practices and preferences in today's clinical laboratory. A report for government regulators, decision makers, and advisors. MLO Med Lab Obs. 1997;29(6):56-57.

6. Anderson NA, Pamphilon DH, Tandy NJ, et al. Comparison of PlateletRich Plasma Collection Using the Haemonetics PCS and Baxter Autopheresis C. Vox Sang. 1991;60(3):155-158.

7. Boyle MJ, Sibal AK, Ruygrok PN, et al. Hybrid management of an unusual presentation of acute ischaemic ventricular septal defect. $N Z$ Med J. 2008;121(1285):119-122.

8. Yanikkaya-Demirel G. ISO 15189 accreditation: Requirements for quality and competence of medical laboratories, experience of a laboratory II. Clin biochem. 2009;42(4-5):279-283.

9. Ashwood ER, Burtis CA, Tietz NW. Tietz fundamentals of clinical chemistry. Elsevier. 2001. p. 976
10. Westgard JO, Miller WG, Allen K, et al. Statistical Quality Control for Quantitative Measurement Procedures: Principles and Definitions: Approved Guideline. CLSI. 2006.

11. Carey RN. User verification of precision and estimation of bias: approved guideline. Clinical and Laboratory Standards Institute. 2014.

12. Mesfin EA, Taye B, Belay $G$, et al. The status of medical laboratory towards of AFRO-WHO accreditation process in government and private health facilities in Addis Ababa, Ethiopia. Pan Afr Med J. 2015:22.136.

13. Ndongmo CB. Clinical laboratory diagnostics in Africa. In African Technology Development Forum Journal. 2005;2(3):21-22.

14. Tamomh AG, Suliman MA, Cedric KK, et al. The role of two parasitological staining techniques in diagnosis of cryptosporidiosis among diarrheic patient's admitted to Kosti teaching hospital, White Nile state, Sudan. MOJ Public Health. 2018;7(2):54-57.

15. Levey S, Jennings ER The use of control charts in the clinical laboratory. Am J Clin Pathol. 1950;20(11):1059-1066.

16. Chosewood LC. Biosafety in microbiological and biomedical laboratories. Diane Publishing. 2007.

17. Sharma SP, Anjankar AP, Kale A. Comparison of glucose levels using glucometer and GOD-POD Method in diabetic patients. Int $J$ Clin Biochem. 2017;4(1):6-10.

18. Neumaier M, Braun A, Gessner R, et al. Experiences with external quality assessment (EQA) in molecular diagnostics in clinical laboratories in Germany. Working Group of the German Societies for Clinical Chemistry (DGKC) and Laboratory Medicine (DGLM). Clin Chem Lab Med. 2000;38(2):161-163.

19. Afrifa J. Quality Control Assessment in the Clinical Chemistry Laboratory-A Ghanaian Study. Doctoral dissertation, Quality Control Assessment in the Clinical Chemistry Laboratory-A Ghanaian Study. 2012.

20. Sutcliffe H. Understanding the NMC code of conduct: a student perspective. Nurs Stand. 2011;25(52):35-39.

21. Westgard JO. Managing quality vs. measuring uncertainty in the medical laboratory. Clin Chem Lab Med. 2010;48(1):31-40.

22. Singh B, Goswami B, Gupta VK, et al. Application of sigma metrics for the assessment of quality assurance in clinical biochemistry laboratory in India: a pilot study. Indian J Clin Biochem. 2011;26(2):131-135.

23. Westgard JO, Westgard SA. The quality of laboratory testing today: an assessment of sigma metrics for analytic quality using performance data from proficiency testing surveys and the CLIA criteria for acceptable performance. Am J Clin Pathol. 2006;125(3):343-354. 